\title{
Thyroglobulin Antibodies as a Prognostic Factor in Papillary Thyroid Carcinoma Patients with Indeterminate Response After Initial Therapy
}

Authors

Giullia Menuci Chianca Landenberger, Marianna Lins de Souza Salerno, Lenara Golbert, Erika Laurini de Souza Meyer

\begin{abstract}
Affiliation
Endocrine Division, Irmandade da Santa Casa de Misericórdia de Porto Alegre, Porto Alegre, RS, Brazil
\end{abstract}

Key words

dynamic risk stratification, initial response to therapy, recurrence

received 26.06.2020

accepted after revision $\quad 25.07 .2020$

published online $\quad 04.09 .2020$

Bibliography

Horm Metab Res 2021; 53: 94-99

DOI $10.1055 / a-1232-4575$

ISSN 0018-5043

(c) 2020. Thieme. All rights reserved.

Georg Thieme Verlag KG, Rüdigerstraße 14,

70469 Stuttgart, Germany

Correspondence

Erika Laurini de Souza Meyer MD, PhD

Department of Internal Medicine, Universidade Federal de

Ciências da Saúde de Porto Alegre - UFCSPA

Rua Sarmento Leite, 245

Porto Alegre

CEP 90050-170, RS

Brazil

Tel.: + 555133303400 , Fax: + 555133303400

erikam@ufcspa.edu.br

\section{ABSTRACT}

The clinical outcome of papillary thyroid carcinoma (PTC) patients with an indeterminate response after initial therapy is reported to be intermediate, between incomplete and excellent responses. This study evaluated the outcomes of PTC patients with indeterminate response after initial therapy. It was further determined whether the indeterminate findings predicted outcomes more precisely. Patients were further classified into 3 groups based on risk of structural persistence/recurrence: $\mathrm{Tg}$ group: detectable thyroglobulin, negative antithyroglobulin antibody, regardless nonspecific imaging findings; TgAb group: positive antithyroglobulin antibody, regardless thyroglobulin levels and nonspecific imaging findings, and Image group: nonspecific findings on neck ultrasonography or faint uptake in the thyroid bed on whole-body scan, undetectable thyroglobulin and negative antithyroglobulin antibody. Sixty-six patients aged $44.1 \pm 12.7$ years were studied, of whom 58 (87.9\%) were females. All patients underwent total thyroidectomy, and 52 patients $(78.8 \%$ ) received radioiodine. After 5.7 years ( $\mathrm{P} 25-75$ 2.6-9.75 years) of follow-up, most patients (89.4\%) were reclassified as having an excellent response or remained in the indeterminate response to therapy. Structural recurrence/persistence disease was detected in 7 (10.6\%) patients. The persistence/recurrence rate in groups were as follow: $\mathrm{Tg}, 2.63 \%$; TgAb, $31.25 \%$; Image, $8.3 \%(\mathrm{p}=0.007)$. The 10 -years disease-free survival rate in the $\mathrm{TgAb}$ group was significantly reduced $(p=0.022)$. Our results suggest that patients with PTC and indeterminate response due to positive serum antithyroglobulin antibody have more risk of development of structural disease. These findings suggest a more individualized follow-up strategy for patients with an indeterminate response.

\section{Introduction}

Although initial risk stratification provides important prognostic information that can allow individualized management recommendations, clinicians have long recognized that dynamic risk-stratification scheme is recommended over time as a function of response to initial therapy and biological behavior of the papillary thyroid cancer (PTC) [1-4]. An indeterminate response is nonspecific biochemical or structural findings that cannot be confidently classified as either benign or malignant [2,3]. The clinical outcome of patients with an indeterminate response is reported to be interme- diate, between incomplete and excellent responses, and the structural persistence/recurrence rate around 13-20\% [3, 5].

Identifying patients at greater risk of adverse outcomes is an important step in the management of PTC patients. Since PTC is known to be usually an indolent neoplasia, the "over follow-up effect" with unnecessary surveillance, diagnostic tests, and medical appointments is a concern [6, 7]. The American Thyroid Association (ATA) guidelines also outline the management implications for patients with an indeterminate response recommending continuous observation with appropriate serial imaging and serum thy- 
roglobulin ( $\mathrm{Tg}$ ) and thyroglobulin antibodies ( $\mathrm{TgAb}$ ) [3]. However, these recommendations are general and nonspecific for this group of PTC patients. Recent studies have demonstrated that patients with indeterminate stimulated $\mathrm{Tg}$ at 12-24 months after initial treatment have more risk of structural disease than patients with positive results for TgAb or nonspecific imaging findings [8]. The objective of the present study is to evaluate the clinical outcome of patients with an indeterminate response in a tertiary, university-based hospital and in addition, to assess whether indeterminate clinical findings in the initial evaluation post-therapy may affect the rates of recurrent/persistent disease.

\section{Patients and Methods}

\section{Study population and design}

We retrospectively reviewed the medical records of Papillary Thyroid Carcinoma (PTC) patients followed at the Endocrine Division of Irmandade da Santa Casa de Misericórdia de Porto Alegre, a tertiary care, university teaching hospital in Southern Brazil. The study was approved by the ethics committees of the institutions (CAAE: 1 . 555.870 and 1.600.048). Following total thyroidectomy (TT) with or without radioactive iodine (RAI) therapy, PTC patients were identified as having an indeterminate response to therapy within the first 6-24 months after initial treatment. An indeterminate response was defined as any of the following findings: (i) basal thyroglobulin $(\mathrm{bTg}) \geq 0.2$ to $<1 \mathrm{ng} / \mathrm{ml}$ or stimulated $\mathrm{Tg}(\mathrm{sTg}) \geq 1$ to $<10 \mathrm{ng} / \mathrm{ml}$ (for patients treated with RAI) and bTg $\geq 0.2$ to $<5 \mathrm{ng} / \mathrm{ml}$ or $s T g \geq 2$ to $<10 \mathrm{ng} / \mathrm{ml}$ (for patients without RAl therapy); (ii) stable or declining TgAb or (iii) nonspecific findings on neck US or faint uptake in the thyroid bed on diagnostic whole-body scan (DxWBS) [3]. Patients with known structural disease after initial treatment were excluded.

\section{Follow-up protocol}

Our treatment protocol is according to the ATA guideline and consists of performing TT, administering or not an ablative or therapeutic dose of RAI and levothyroxine suppression therapy according to the initial risk assessment [3]. Follow-up duration was defined as the time between the TT and the last medical visit to the clinic. Structural disease was defined based on imaging, cytology or histology results, and/or unequivocal ectopic uptake (excluding false-positive results) on post-therapy DxWBS or FDG-PET/CT.

\section{Risk stratification and outcomes}

All patients were classified according to the ATA 2015, into low, intermediate, or high risk of recurrence [3]. Additionally, they were staged according to the American Joint Committee on Cancer and International Union Against Cancer (TNM staging $8^{\text {th }}$ edition) [9]. As patients were followed up, response to therapy was dynamically defined until last follow-up visit as excellent, indeterminate, biochemical incomplete and structural incomplete, according to ATA 2015 guidelines [3]. When evaluating the initial response therapy, PTC patients were further classified into 3 groups in order to specify the indeterminate group according to recurrence. Tg group: detectable $\mathrm{Tg}$ and negative $\mathrm{TgAb}$, regardless nonspecific imaging findings; $T g A b$ group: positive $\mathrm{TgAb}$, regardless $\mathrm{Tg}$ levels and nonspecif- ic imaging findings; Image group: nonspecific findings on neck US or faint uptake in the thyroid bed on DxWBS, undetectable Tg and negative TgAb. For the purposes of patient enrollment and the analysis, the results of each $\mathrm{TgAb}$ assay were recorded simply as "positive" or "negative" based on a titer above or below the cut-off of the specific assay. We considered TgAb positive with any value different from negative value according to the method. The primary outcome of this study was structural disease. Disease free survival (DFS) was considered the time from initial surgery to last follow-up or structural disease detected.

\section{Laboratorial analysis}

Serum Tg measurements were performed using immunoradiometric assays: radioimmunoassay (until 2000); electrochemiluminescence (from 2000 to 2011), and chemiluminescence (from 2011 until the present), with functional sensitivities from 0.5 to the current $0.1 \mathrm{ng} / \mathrm{ml}$. Serum TgAb were measured using the agglutination method until 2001, electrochemiluminescence until 2003 and by chemiluminescence from 2004 until the present. Serum TSH levels were measured with a chemiluminescence assay.

\section{Statistical analysis}

Clinical and laboratory data are described as the mean \pm standard deviation (SD) or median and percentiles 25 and 75 (P25-75) for continuous variables and absolute numbers and percentages for categorical variables. To determine the association between categorical variables, the chi-square test was used. The DFS curve was plotted using KaplanMeier method and the log-rank test was used to determine their significance. Data analysis was performed using Statistical Package for Social Sciences (SPSS) software, version 25.0 (IBM Corp., Armonk, NY). A p-value of $<0.05$ was considered statistically significant.

\section{Results}

\section{Baseline characteristics of PTC patients with indeterminate response to therapy}

Sixty-six PTC patients were included in this study. In the general evaluation of the sample, the mean age at diagnosis was $44.1 \pm 12.1$ years, 58 patients ( $87.9 \%$ ) were women and 50 patients $(75.8 \%)$ were $<55$ years of age. All patients underwent TT \pm lymph node dissection and 52 patients $(78.8 \%$ ) received ablative or therapeutic dose of RAI. Clinical and oncological characteristics of these patients according to the indeterminate group are described in - Table 1. There were no significant differences in prognostic clinical features between groups.

\section{Disease status of PTC patients with indeterminate response at last evaluation}

- Figure 1 shows the outcomes of PTC patients according to the dynamic risk stratification. After a median follow-up of 5.7 years (2.69.75 years), 32 out of 66 (48.5\%) of indeterminate patients were reclassified as excellent response. Among them, $71.9 \%$ were without additional therapy. In fact, the majority of patients (59/66; 89.4\%) with an indeterminate response to therapy remain disease free during prolonged follow-up. It was observed that among patients with excellent or indeterminate response at final evaluation, only 2 pa- 
- Table 1 Characteristics of patients with papillary thyroid carcinoma and indeterminate response after initial therapy according to the indeterminate group.

\begin{tabular}{|c|c|c|c|c|}
\hline & $\mathrm{Tg}$ & $\operatorname{TgAb}$ & Image & p-Value \\
\hline Female sex - n (\%) & 32 (84.2) & $15(93.8)$ & $11(91.7)$ & 0.560 \\
\hline Age at diagnosis in years - mean $( \pm S D)$ & $42.8( \pm 12.8)$ & $47.4( \pm 14.5)$ & $44.3( \pm 10.3)$ & 0.491 \\
\hline$<55$ years at diagnosis $-\mathrm{n}(\%)$ & 30 (78.9) & $10(62.5)$ & $10(83.3)$ & 0.347 \\
\hline Primary tumor size, cm - median (P25-75) & $1.75(0.68-2.58)$ & $2.40(1.08-3.95)$ & $1.25(0.73-2.08)$ & 0.495 \\
\hline Microcarcinoma - n (\%) & $13(34.2)$ & $5(31.3)$ & $4(33.3)$ & 0.978 \\
\hline \multicolumn{5}{|l|}{ AJCC $8^{\text {th }}$ Clinical Stage $-\mathrm{n}(\%)$} \\
\hline Stage I & $34(89.5)$ & $15(93.8)$ & $12(100)$ & 0.473 \\
\hline Stage II & $4(10.5)$ & $1(6.3)$ & 0 & \\
\hline \multicolumn{5}{|l|}{ ATA 2015 Risk Stratification - n (\%) } \\
\hline Low & $11(28.9)$ & $7(43.8)$ & $7(58.3)$ & 0.295 \\
\hline Intermediate & $19(50)$ & $8(50)$ & $4(33.3)$ & \\
\hline High & $8(21.1)$ & $1(6.3)$ & $1(8.3)$ & \\
\hline Total thyroidectomy - n (\%) & $20(52.6)$ & $10(62.5)$ & $7(58.3)$ & 0.788 \\
\hline Total thyroidectomy with neck dissection - $\mathrm{n}(\%)$ & $18(47.4)$ & $6(37.5)$ & $5(41.7)$ & \\
\hline Patients who received RAI - n (\%) & 30 (78.9) & $13(81.2)$ & $9(75)$ & 0.789 \\
\hline Total RAI dose, mCi - median (P25-75) & $114(100-162)$ & $100(30-275)$ & $100(40-125)$ & 0.922 \\
\hline Follow-up time, years - median (P25-75) & $5.65(2.78-8.88)$ & $7(3.23-10.25)$ & $3.90(2.23-7.78)$ & 0.348 \\
\hline
\end{tabular}

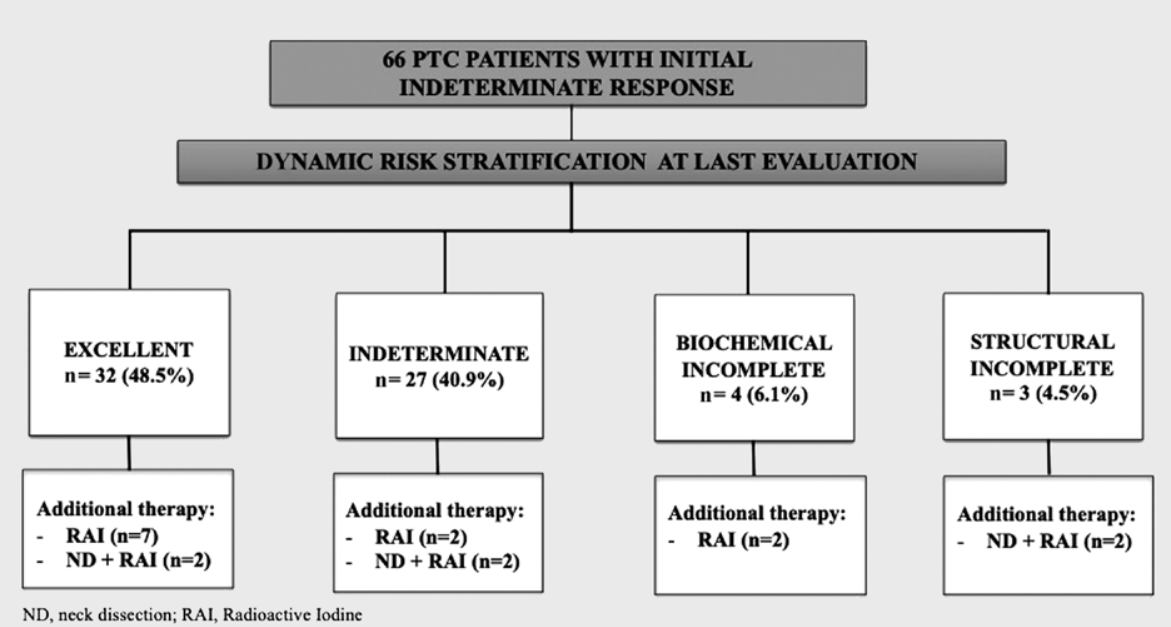

Fig. 1 Follow-up outcomes, according to the dynamic risk stratification (DRS). PTC: Papillary thyroid carcinoma.

tients (6.3\%) and 4 patients (14.8\%), respectively, were high recurrence risk. No low-risk patient showed evidence of disease.

\section{Clinicopathologic features of patients with structural disease}

Structural persistent/recurrent disease was detected in 7 (10.6\%) patients during follow-up. The median time to detection of structural disease from the initial thyroid surgery was 7.2 years (min 4.1
- max 11.8 years). All patients had cervical disease, and one patient had concomitant lung metastasis. Five patients (71.4\%) were classified as intermediate risk of recurrence and 2 patients (28.6\%) as high risk. Interestingly, 5 of 7 patients $(71.4 \%$ ) had positive thyroglobulin antibodies at first 24 months after initial therapy, among them, 2 patients also had nonspecific findings on neck US. 


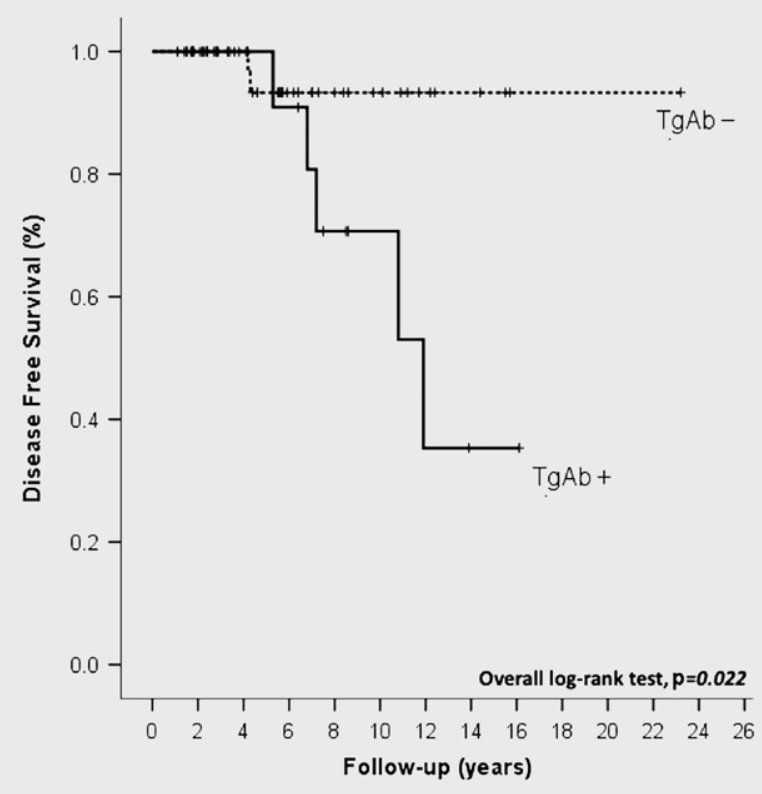

- Fig. 2 Disease-free survival rates in patients with papillary thyroid carcinoma who have indeterminate response according to positive antithyroglobulin antibody $(\mathrm{TgAb}+)$ and negative antithyroglobulin antibody (TgAb-). Overall log-rank test, $\mathrm{p}=0.022$.

\section{Structural disease and disease-free survival (DFS) of PTC patients according to indeterminate response group}

The persistence/recurrence rate in the $\operatorname{Tg} A b$ group was significantly higher than in the $\mathrm{Tg}$ and image groups, as follows: $\mathrm{Tg}$ group (1/38), 2.63\%; TgAb group (5/16), 31.25\%; Image group (1/12), $8.3 \%$ $(p=0.007)$. To further explore a possible role of TgAb positivity on disease outcome, we compared the DFS rates in patients with positive $\operatorname{TgAb}(\operatorname{TgAb}+)(n=16)$ and negative $\operatorname{TgAb}(\operatorname{TgAb}-)(n=50)$, regardless $\mathrm{Tg}$ levels and nonspecific imaging findings. The 10-year DFS rate in group of patients with increase of TgAb levels was significantly reduced $(p=0.022)(\vee$ Fig. 2$)$. The 10 -year DFS rates of patients in the TgAb + and TgAb- group were, $70.7 \%$ and $93.3 \%$, respectively.

\section{Discussion}

This study demonstrated that most PTC patients with an indeterminate response after initial therapy remain disease-free after prolonged follow-up. Also, it was shown that the presence of $\mathrm{TgAb}$, regardless of nonspecific imaging findings is a potential prognostic factor to predict disease status in these patients.

In our sample, the majority of patients (89.4\%) were reclassified as having an excellent response or remained in the indeterminate response therapy after follow-up. In fact, in a cohort of 588 DTC patients treated with TT and RAI therapy, the risk of having persistent or recurrent disease was significantly higher in patients having an incomplete response to therapy (96\%) than in patients having an excellent (4\%) or indeterminate response to therapy (13\%) [4]. In our study, structural persistent/recurrent disease was detected in $10.6 \%$ of patients, less frequent than other studies that demonstrate rates of $13-20 \%$ in a longer follow-up $[4,5,8]$. Of note, the majority of the patients in the present study were stage I and $85 \%$ of them were low or intermediate initial risk of recurrence.

We observed that PTC patients with positive TgAb, at first 24 months after initial therapy have more recurrence/persistent structural disease. Between patients with structural disease, $71.4 \%$ presented positive TgAb at initial treatment response. In addition, the frequency of ATA initial risk and TNM stage among the groups seem not to contribute to this outcome, since there were no differences between groups.

Thyroglobulin antibodies are present in about $14 \%$ of patients with PTC in preoperative, but fall in the first year after effective initial therapy, especially with RAl therapy $[10,11]$. Previous studies have demonstrated that PTC patients with positive serum $\mathrm{TgAb}$ titer during the first year after treatment were more likely to have persistent/recurrent disease than those who were consistently TgAb-negative. Negative titers at 1 year may be associated with more favorable outcomes [12,13]. In our study, most patients received RAI therapy, and these findings suggest that patients with persistent elevated TgAb levels in the first 24 months after primary therapy may have more chance of disease. In this way, monitoring TgAb titers can provide surrogate information on disease recurrence, and declining titers are usually associated with remission $[12,13]$.

The clinical importance of low but detectable basal or stimulated levels of $\mathrm{Tg}$ is unclear. In contrast of our results, a recent study has shown more prevalence of structural disease in patients with indeterminate stimulated Tg levels [8]. However, in the study cited above, the proportion of stage I disease or low-risk patients was significantly smaller in the Tg group than in the TgAb or image groups. Furthermore, Tg levels were evaluated regardless the presence of $\mathrm{TgAb}$, that can interfere with measurements of serum $\mathrm{Tg}$. In fact, not all patients in our study were initially evaluated by stimulated $\mathrm{Tg}(\mathrm{sTg}) ; 22$ of 38 patients ( $57.9 \%$ ) had sTg, with median of $4.06 \mathrm{ng} / \mathrm{ml}$ (data not shown). The availability of ultrasensitive $\mathrm{Tg}$ assays seems to remove the need for routine sTg determinations $[3,14]$. The role of basal and/or stimulated Tg in evaluation of therapy in patients with indeterminate response is not well established. Based on STg, a change in category occurred in half the patients with an initial indeterminate response, $44.4 \%$ to excellent response and $5.5 \%$ to biochemical incomplete response and others remained in same category [15]. Interestingly, some studies have demonstrated that if sTg is done 5 years after the initial treatment, the vast majority of responses originally classified as indeterminate (up to $98 \%$ ) can be reclassified as excellent response [16]. In addition, patients with indeterminate response, rising values of unstimulated $\mathrm{Tg}$, independently from the basal levels, may be useful to identify patients with progressive disease [17]. Therefore, following the curve of basal $\mathrm{Tg}$ seems to be a suitable laboratory tool in selected cases of indeterminate response [7].

In this study, $8.3 \%$ of patients in the image group had cervical structural disease during follow-up. Nonspecific lesions can be difficult to distinguish between recurrent thyroid cancer and non-recurrent benign lesions with neck US alone [18-22]. Recent studies have proposed to restrict US to patients with basal $\mathrm{Tg} \geq 1 \mathrm{ng} / \mathrm{ml}$ after treatment with RAI because this imaging method revealed 
disease in a small number of patients with basal $\mathrm{Tg}<1 \mathrm{ng} / \mathrm{ml}$ $[21,22]$. In fact, it is very important the routine neck US evaluation after thyroid surgery in the setting of increased $\mathrm{Tg}$ levels or presence of increasing $\mathrm{TgAb}$ levels [3, 22, 23]. Of note, the European Thyroid Association guidelines identify US characteristics that can be used to distinguish indeterminate from suspicious findings in neck US, being useful in evaluation of PTC patients with indeterminate $\mathrm{Tg}$ and/or presence of TgAb [24].

There are some limitations to this study. It is a retrospective design study in a small sample, especially $\mathrm{TgAb}$ positive patients. Because the cohort was composed of patients diagnosed over a period of 20 years, baseline $\mathrm{Tg}$ levels were measured with different assays. The fact that all assays used in this cohort had functional sensitivities of $1 \mathrm{ng} / \mathrm{ml}$ or less allowed us to retrospectively analyze the results using the cut-off value recommended by the ATA [3]. Another possible limitation is that there was no standardization of US evaluation to address lymph node disease. However, this limitation may be partially mitigated by the fact that all ultrasound reports were reviewed. Despite these limitations, this study included patients seen at the same institution, were evaluated by the same follow-up and investigation protocol.

In conclusion, the majority of patients with an indeterminate response to therapy remain disease-free during prolonged follow-up. Our results suggest that PTC patients with indeterminate response after initial therapy by the presence of positive serum $\mathrm{TgAb}$ have more risk of development of structural disease in the long term. Prospective studies with a larger number of patients and long-term follow-up are needed to clarify the potential role of positive serum $\mathrm{TgAb}$ in the prediction of PTC.

\section{Acknowledgements}

We wish to thank the surgeons of our Hospital (Dr. Virgilio Zanella and Dr. Luis Felipe Osowski) for surgical management of our patients, Dr. Marinez Bizarro Barra for anatomopathological analysis and Dr. Kamille Guidolin for helping in assisting our patients. We are grateful to Cristiane Bündchen for her excellent statistical support.

\section{Conflict of Interest}

The authors declare that they have no conflict of interest.

\section{References}

[1] Vaisman F, Tutlle RM. Clinical assessment and risk stratification in differentiated thyroid cancer. Endocrinol Metab Clin North Am 2019; 48: 99-108

[2] Tuttle RM, Alzahrani AS. Risk stratification in differentiated thyroid carcinoma: From detection to final follow-up. J Clin Endocrinol Metab 2019; 104: 4087-4100

[3] Haugen BR, Alexander EK, Bible KC et al. 2015 American Thyroid Association Management Guidelines for Adult Patients with Thyroid Nodules and Differentiated Thyroid Cancer. Thyroid 2016: 26: 1-133
[4] Tuttle RM, Tala H, Shah J et al. Estimating risk of recurrence in differentiated thyroid cancer after total thyroidectomy and radioactive iodine remnant ablation: Using response to therapy variables to modify the initial risk estimates predicted by the new American Thyroid Association staging system. Thyroid 2010; 20: 1341-1349

[5] Jeon MJ, Kim WG, Park WR et al. Modified dynamic risk stratification for predicting recurrence using the response to initial therapy in patients with differentiated thyroid carcinoma. Eur J Endocrinol 2013; 170: $23-30$

[6] Scheffel RS, Zanella AB, Antunes D et al. Low recurrence rates in a cohort of differentiated thyroid carcinoma patients: A referral center experience. Thyroid 2015; 25: 883-889

[7] Pacini F, Basolo F, Bellantone R et al. Italian consensus on diagnosis and treatment of differentiated thyroid cancer: Joint statements of six Italian societies. J Endocrinol Invest 2018; 41: 849-876

[8] Oh HS, Ahn JH, Song E et al. Individualized follow-up strategy for patients with an indeterminate response to initial therapy for papillary thyroid carcinoma. Thyroid 2019; 29: 209-215

[9] Tuttle RM, Haugen B, Perrier ND. Updated American Joint Committee on Cancer/ Tumor-Node-Metastasis Staging System for Differentiated and Anaplastic Thyroid Cancer ( $8^{\text {th }}$ ed): What Changed and Why? Thyroid 2017; 27: 751-756

[10] Kim WG, Yoon JH, Kim WB et al. Change of serum antithyroglobulin antibody levels is useful for prediction of clinical recurrence in thyroglobulin-negative patients with differentiated thyroid carcinoma. J Clin Endocrinol Metab 2008; 93: 4683-4689

[11] Xu J, Bergren R, Schneider D et al. Thyroglobulin antibody resolution after total thyroidectomy for cancer. J Surg Res 2015; 198: 366-370

[12] Durante C, Tognini S, Montesano T et al. Clinical aggressiveness and long-term outcome in patients with papillary thyroid cancer and circulating anti-thyroglobulin autoantibodies. Thyroid 2014; 24 : 1139-1145

[13] Rosario PW, Carvalho M, Mourão GF, Calsolari M. Comparison of antithyroglobulin antibody concentrations before and after ablation with 131 l as a predictor of structural disease in differentiated thyroid carcinoma patients with undetectable basal thyroglobulin and negative neck ultrasonography. Thyroid 2016; 26: 525-531

[14] Schlumberger M, Hitzel A, Toubert ME et al. Comparison of seven serum thyroglobulin assays in the follow-up of papillary and follicular thyroid cancer patients. J Clin Endocrinol Metab 2007; 92: 2487-2495

[15] Rosario PW, Mourão GF, Calsolari MR. Definition of the response to initial therapy with radioiodine in patients with differentiated thyroid carcinoma: Basal or stimulated thyroglobulin? Horm Metab Res 2019; 51: 634-638

[16] Lamartina L, Montesano T, Trulli F et al. Papillary thyroid carcinomas with biochemical incomplete or indeterminate responses to initial treatment: Repeat stimulated thyroglobulin assay to identify disease-free patients. Endocrine 2016; 54: 467-475

[17] Malandrino P, Tumino D, Russo M et al. Surveillance of patients with differentiated thyroid cancer and indeterminate response: $A$ longitudinal study on basal thyroglobulin trend. J Endocrinol Invest 2019; 42: 1223-1230

[18] Lamartina L, Grani G, Biffoni M et al. Risk stratification of neck lesions detected sonographically during the follow-up of differentiated thyroid cancer. J Clin Endocrinol Metab 2016; 101: 3036-3044

[19] Robenshtok E, Fish S, Bach A et al. Suspicious cervical lymph nodes detected after thyroidectomy for papillary thyroid cancer usually remain stable over years in properly selected patients. J Clin Endocrinol Metab 2012; 97: 2706-2713

[20] Epstein S, McEachern R, Khot R et al. Papillary thyroid carcinoma recurrence: Low yield of neck ultrasound with an undetectable serum thyroglobulin level. J Ultras Med 2018; 37: 2325-2331 
[21] Verburg FA, Mäder U, Giovanella L et al. Low or undetectable basal thyroglobulin levels obviate the need for neck ultrasound in differentiated thyroid cancer patients after total thyroidectomy and 1311 ablation. Thyroid 2018; 28: 722-728

[22] Rosario PW, Mourão GF, Calsolari MR. Repeat ultrasonography in the first years after therapy with radioiodine is not necessary in most patients with papillary thyroid carcinoma when postoperative ultrasonography is negative: A reduction of costs and false-positives. Eur Thyroid J 2019; 8: 41-45
[23] Lamartina L, Grani G, Durante C et al. Follow-up of differenciated thyroid cancer - what should (and what should not) be done. Nature Reviews. Endocrinology 2018; 14: 538-551

[24] Leenhardt L, Erdogan MF, Hegedus L et al. European Thyroid Association guidelines for cervical ultrasound scan and ultrasound-guided techniques in the postoperative management of patients with thyroid cancer. Eur Thyroid J 2013; 2: 147-155 